\title{
Impact analysis of actuator torque degradation on the IRB 120 robot performance using simscape-based model
}

\author{
Le Ngoc Truc ${ }^{1}$, Nguyen Phung Quang ${ }^{2}$, Nguyen Hong Quang ${ }^{3}$ \\ ${ }^{1}$ Hung Yen University of Technology and Education, Hung Yen, Vietnam \\ ${ }^{2}$ Institute for Control Engineering and Automation, Hanoi University of Science and Technology, Hanoi, Vietnam \\ ${ }^{3}$ Thai Nguyen University of Technology, Thai Nguyen City, Vietnam
}

\begin{abstract}
Article Info
Article history:

Received Jul 1, 2020

Revised Apr 9, 2021

Accepted Apr 26, 2021

\section{Keywords:}

Actuator fault

Joint failure

Robot manipulator

Simscape-based model

Torque degradation

ABSTRACT

Actuators in a robot system may become faulty during their life cycle. Locked joints, free-moving joints, and the loss of actuator torque are common faulty types of robot joints where the actuators fail. Locked and free-moving joint issues are addressed by many published articles, whereas the actuator torque loss still opens attractive investigation challenges. The objectives of this study are to classify the loss of robot actuator torque, named actuator torque degradation, into three different cases: Boundary degradation of torque, boundary degradation of torque rate, and proportional degradation of torque, and to analyze their impact on the performance of a typical 6-DOF robot (i.e., the IRB 120 robot). Typically, controllers of robots are not pre-designed specifically for anticipating these faults. To isolate and focus on the impact of only actuator torque degradation faults, all robot parameters are assumed to be known precisely, and a popular closed-loop controller is used to investigate the robot's responses under these faults. By exploiting MATLAB-the reliable simulation environment, a simscape-based quasi-physical model of the robot is built and utilized instead of an actual expensive prototype. The simulation results indicate that the robot responses cannot follow the desired path properly in most fault cases.
\end{abstract}

This is an open access article under the CC BY-SA license.

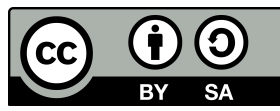

\section{Corresponding Author:}

Nguyen Hong Quang

Thai Nguyen University of Technology

666, 3/2 Street, Tich Luong Ward, Thai Nguyen City 251750, Vietnam

Email: quang.nguyenhong@tnut.edu.vn

\section{INTRODUCTION}

Up to now, robotics have been still receiving the attention of a significant number of researchers and manufacturers because of their great advantages in many fields [1]-[4]. During the robot life cycle with unforeseen occurrences, the operation and performance of robots may be affected by actuator faults. A condition monitoring system using neural-networks and signal analysis techniques is provided in [5] to detect and diagnose the robot joint failures. Many robots are designed with redundant kinematics and/or actuators to get ready for this problem. For 7-DOF space manipulators with one redundant joint, the effective solutions to resolve the inverse kinematics are presented when an arbitrary joint is locked [6]. A method is proposed to identify the optimal kinematic design of a redundant robot manipulator for having a high fault-tolerant capability in case of single locked joint events [7]. Several suitable strategies are applied to deal with locked joint faults [8]-[10] or free-swinging joint faults [11] occurring in redundant robots. The techniques in estimating surfaces of self-motion, analyzing and determining the required joint constraints are proposed in [8] to guarantee the 
reachability of robots with a specified task. With pick-and-place tasks, a kinematic fault-tolerant approach to find an obstacle avoidance path in the working space are presented in [9]. When a redundant serial robot suffers locked joint faults, a generalized framework of optimal velocity jump mapping is introduced for minimizing the end-effector velocity jump [10]. For anticipating free-swinging joint problem, the study in [11] provides a method using fault tolerance as an extra criterion to find the best configuration of robot links in the point view of slow movements before a joint fault event. For robots without redundancy, several efficient methods to improve the performance of robot manipulators have been introduced [12]-[14]. The free-swinging joint faults are dealt by a fault detection strategy and a robust adaptive control procedure with three modes (regulating faulty joints, braking faulty joints, and controling active joints) by considering robots as underactuated robots [12]. By redefining the reachable working space in the event of single locked joint failures and combining with the desired end-effector performance, a fault-tolerant path method for space manipulators is provided to obtain the reasonable path [13], [14]. However, most of those studies mention only in the locked joint and/or free-swinging joint faults. In reality, robot actuators can be subject to torque degradation faults. Therefore, this issue needs to be analyzed and to propose proper solutions for enhancing system behavior.

There are currently numerous published researches relating to the general or specific fault issues existing in systems, input signals, and actuators. Polycarpou [15], a learning methodology is developed for failure compensation of a special class of multiple-input multiple-output nonlinear systems with a particular condition, which is based on the idea of a corrective control algorithm made active when faults happen. However, different categories for types of failures, especially input failures, are not classified. Xing et al. [16], a new event-triggered control fashion for a class of uncertainty nonlinear systems suffering unknown actuator faults is presented. A novel adaptive failure compensation control methodology is nominated for nonlinear systems with parametric rigorous feedback [17]. Yang et al. [18], propose a new adaptive failure tolerant controller design for unknown nonlinear systems with multiple actuators. Nevertheless, those studies in [15]-[18] consider general nonlinear systems. A comprehensive classification of actuator losses needs to be figured out for a specific multibody mechanical system, especially a serial robot manipulator. Moreover, their impacts on the system performance have certain differences and still receive considerable interest. Freddi et al. [19], a kinematic failure tolerant control method for robotic arms affected by torque failures is provided. Experiments are conducted in two sorts of actuator faults, which are additive time-varying fault and additive constant fault. In [20], [21], model-based failure detection and isolation methodology for robotic manipulators using an adaptive nonlinear observer designed to handle actuator and sensor faults. A fault detection and isolation technique for the robot actuator effectiveness using Super-Twisting second order sliding mode observers is presented in [22]. The study in [23] shows an actuator failure detection and an adaptive control approach for flexible joint robots with uncertainty parameters and multiple actuator failures. A method to detect and compensate the proportional loss of torque of a 2-DOF robot arm is addressed in [24]. Therein, unknown proportional coefficients of torque are combined with other model parameters, and then an adaptive law is synthesized to update uncertainties and to maintain the robot responses.

In the above investigation, actuators operating in some fault modes are modeled. Free-swinging joint, locked joint, proportional degradation of actuator torque faults, and another specific kind of actuator failures that torque is stuck at an unknown constant value; all of them are mentioned as well. But the two other essential actuator faults, which are the boundary degradation of torque and the boundary degradation of torque rate, have not been analyzed yet. On the other hand, the free-swinging joint fault is just a case of proportional degradation of torque when the actual generated torque is zero, and the joint is unblocked. From the point of system control view, the locked joint fault can also be considered as a particular case of proportional degradation of torque when the actuator torque degrades to zero, and the joint is blocked by either the integrated automatic brake or mechanical causes. Consequently, in this case, the DOF number of a robot and its working range will be reduced.

This paper aims to clarify torque degradation fault types of robot actuators and evaluate their impact on system responses separately through numerical simulation using a quasi-physical robot model. The actuator torque degradation faults of robots are divided into three types: Boundary degradation of torque (BDT), boundary degradation of torque rate (BDTR), and proportional degradation of torque (PDT). Torque being stuck at an unknown constant value almost does not exist in real robots; therefore, in this study, we do not concentrate on that rare state. The ABB IRB 120 robot, which is a typical 6-DOF industrial robot, is put into the investigation. To focus on only the impact of actuator torque degradation faults, all robot parameters are assumed to be known and perfect. A computed torque controller pre-designed in regular operation can be applied to 
evaluate the impact of torque degradation on robot performance. Moreover, the simscape-based quasi-physical model of the IRB 120 robot is used for the accuracy and reliability of simulation. In the following parts of this paper, section 2 describes both the dynamic modeling of the IRB 120 robot and its simscape-based model. Section 3 presents and formulates three types of actuator torque degradation faults: BDT, BDTR, and PDT in detail. Section 4 investigates the robot responses in the presence of degraded torque via various significant simulations to analyze their impact. Some critical remarks are finally noted in section 5.

\section{MODELING OF THE IRB 120 ROBOT}

\subsection{Mathematical dynamic modeling of the robot}

The configuration, dimensions, and D-H parameters of the ABB IRB 120 robot which has 6 revolute joints are described in Figure 1 and Table 1 [25]. The mathematical model of the robot dynamics with nonfriction is (1):

$$
M \ddot{q}+C \dot{q}+g=\tau
$$

where $q \in R^{6}$ is the angular variable vector, $M:=M(q) \in R^{6 \times 6}$ is the generalized inertia matrix, $C:=$ $C(q, \dot{q}) \in R^{6 \times 6}$ is the Coriolis/centrifugal matrix, $g:=g(q) \in R^{6}$ is the gravity term vector, $\tau \in R^{6}$ is the actual torque vector generated by actuators. Based on the results presented in [26], the vector/matrix parameters in (1) can be calculated by the following formulas (2), (3), (4):

$$
\begin{gathered}
M=\sum_{i=1}^{6}\left(m_{i}\left(J_{T_{i}}^{0}\right)^{T} J_{T_{i}}^{0}+J_{R_{i}}^{T} I_{i} J_{R_{i}}\right) \\
C=\frac{1}{2}\left[\frac{\partial M}{\partial q}\left(1_{6} \otimes \dot{q}\right)-\left(\frac{\partial M}{\partial q}\left(\dot{q} \otimes 1_{6}\right)\right)^{T}+\frac{\partial M}{\partial q}\left(\dot{q} \otimes 1_{6}\right)\right] \\
g=\left(\frac{\partial P}{\partial q}\right)^{T}
\end{gathered}
$$

where $m_{i}$ is the link mass; $I_{i} \in R^{3 \times 3}$ is the link inertia tensor represented in the frame which is attached at the link centroid and parallel to frame $i ; J_{R_{i}}$ and $J_{T_{i}}^{0} \in R^{3 \times 6}$ are rotational and translational Jacobian matrices expressed in frame $i$ and the base frame, respectively; $\mathbf{1}_{6}$ is the 6-by-6 identity matrix; $\otimes$ is the Kronecker product operator; $P$ is the total potential energy of the robot. All above parameters will be achieved from official robot 3D CAD technical files by exploiting professional CAD software.

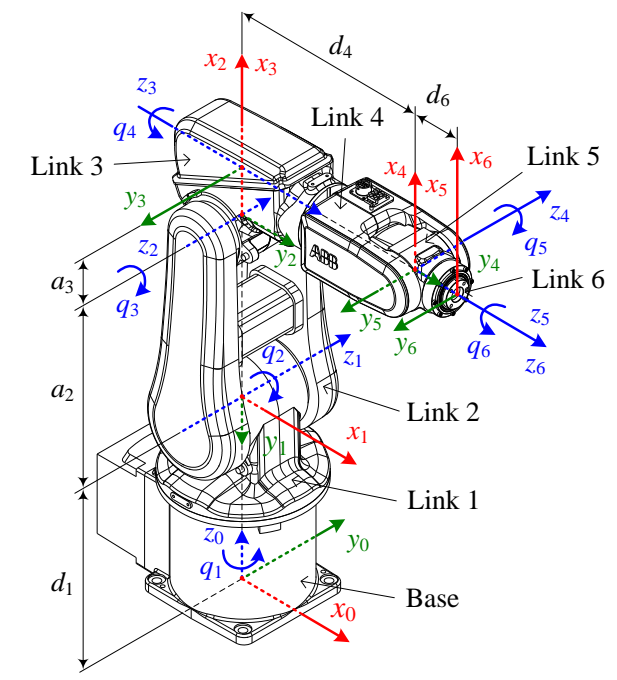

Figure 1. Initial configuration and attached frames of the IRB 120 robot 
Table 1. D-H parameters of the IRB 120 robot

\begin{tabular}{lllll}
\hline Joint $i$ & $\theta_{i}(\mathrm{rad})$ & $d_{i}(\mathrm{~m})$ & $a_{i}(\mathrm{~m})$ & $\alpha_{i}(\mathrm{rad})$ \\
\hline 1 & $q_{1}$ & $d_{1}=0.290$ & $a_{1}=0$ & $\alpha_{1}=-\frac{\pi}{2}$ \\
2 & $q_{2}-\frac{\pi}{2}$ & $d_{2}=0$ & $a_{2}=0.270$ & $\alpha_{2}=0$ \\
3 & $q_{3}$ & $d_{3}=0$ & $a_{3}=0.070$ & $\alpha_{3}=-\frac{\pi}{2}$ \\
4 & $q_{4}$ & $d_{4}=0.302$ & $a_{4}=0$ & $\alpha_{4}=\frac{\pi}{2}$ \\
5 & $q_{5}$ & $d_{5}=0$ & $a_{5}=0$ & $\alpha_{5}=-\frac{\pi}{2}$ \\
6 & $q_{6}$ & $d_{6}=0.072$ & $a_{6}=0$ & $\alpha_{6}=0$ \\
\hline
\end{tabular}

\subsection{Simscape-based quasi-physical modeling of the robot}

The torque degradation of robot actuators can happen suddenly or progressively in reality and robot systems should be investigated and prepared well for facing this fault. Setting up and conducting a practical experiment will always be excellent. However, it is not easy to create an exact fault-ready-made prototype for the IRB 120 robot or to approach a real industrial robot being torque-degraded for investigating. Hence, a quasiphysical model of the robot is exploited using simscape multibody to analyze the problem as well as its impact on the robot's performance. Then some valuable comments and evaluations can support the next work in failure diagnosis and the design of fault-tolerant control strategies more effectively. MATLAB/simscape multibody provides a powerful quasi-physical simulation environment for multi-component mechanical systems such as robots, multibody systems, hydraulic and pneumatic systems. The simscape-based approach has been exploited successfully in several studies, e.g., [27]-[29]. Simscape multibody gives blocks representing links, joints, sensors, and torque/force components that make the simscape-based model conformable to the actual robot. With a geometric structure of link, simscape multibody allows users to draw with simple blocks. However, you can use professional CAD software to design bodies (autodesk inventor, solidWorks, and onshape, for example), and then MATLAB/simscape multibody can use these CAD files to generate the system model. Based on the CAD models provided by ABB Inc. for the IRB 120 robot [30], we exploit autodesk inventor to explore the parameters of the robot and to construct its 3D assembly model in Figure 2. The approximated values of mass $m_{i}(\mathrm{~kg})$, link centroids $r_{C_{i}}(\mathrm{~m})$, inertia tensors $I_{i}\left(\mathrm{kgm}^{2}\right)$ can be achieved by performing the physics analysis method of Autodesk Inventor and are described in (5). Autodesk inventor exports a robot CAD assembly file and a set of geometry files that can be imported into MATLAB simscape multibody to support the generation of the simscape-based quasi-physical model of the robot with the same physics description as shown in Figure 3.

Base: $m_{0}=8.659$

Link 1: $m_{1}=4.248, r_{C_{1}}=[0,0.054,0]^{T}, I_{1}=\left[\begin{array}{ccc}19.7 & 0 & 0 \\ 0 & 14.5 & 0 \\ 0 & 0 & 19.9\end{array}\right] 10^{-3}$

Link 2: $m_{2}=5.412, r_{C_{2}}=[-0.169,0,0]^{T}, I_{2}=\left[\begin{array}{ccc}35.9 & 0 & 0 \\ 0 & 83.5 & 0 \\ 0 & 0 & 57.6\end{array}\right] 10^{-3}$

Link 3: $m_{3}=4.077, r_{C_{3}}=\left[\begin{array}{lll}-0.012, & 0,0.023\end{array}\right]^{T}, I_{3}=\left[\begin{array}{ccc}17.6 & 0 & -2 \\ 0 & 23.1 & 0 \\ -2 & 0 & 11.6\end{array}\right] 10^{-3}$

Link 4: $m_{4}=1.832, r_{C_{4}}=[0,-0.007,0]^{T}, I_{4}=\left[\begin{array}{ccc}7.2 & 0 & 0 \\ 0 & 3.9 & 0 \\ 0 & 0 & 5.6\end{array}\right] 10^{-3}$

Link 5: $m_{5}=0.755, r_{C_{5}}=[0,0,0]^{T}, I_{5}=\left[\begin{array}{ccc}1.1 & 0 & 0 \\ 0 & 1.2 & 0 \\ 0 & 0 & 0.6\end{array}\right] 10^{-3}$

Link 6: $m_{6}=0.019, r_{C_{6}}=[0,0,-0.007]^{T}, I_{6}=\left[\begin{array}{ccc}2.3 & 0 & 0 \\ 0 & 2.3 & 0 \\ 0 & 0 & 4.1\end{array}\right] 10^{-6}$ 


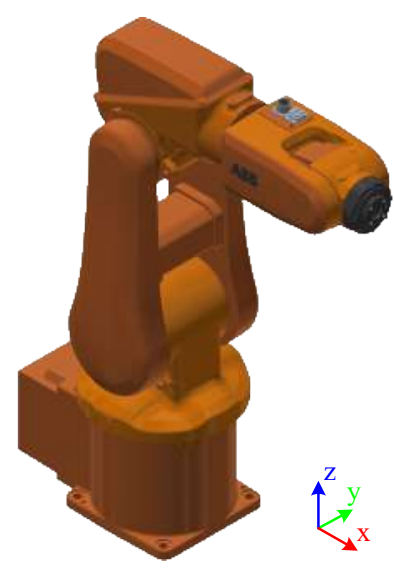

Figure 2. 3D CAD assembly model of the IRB 120 robot constructed by autodesk inventor

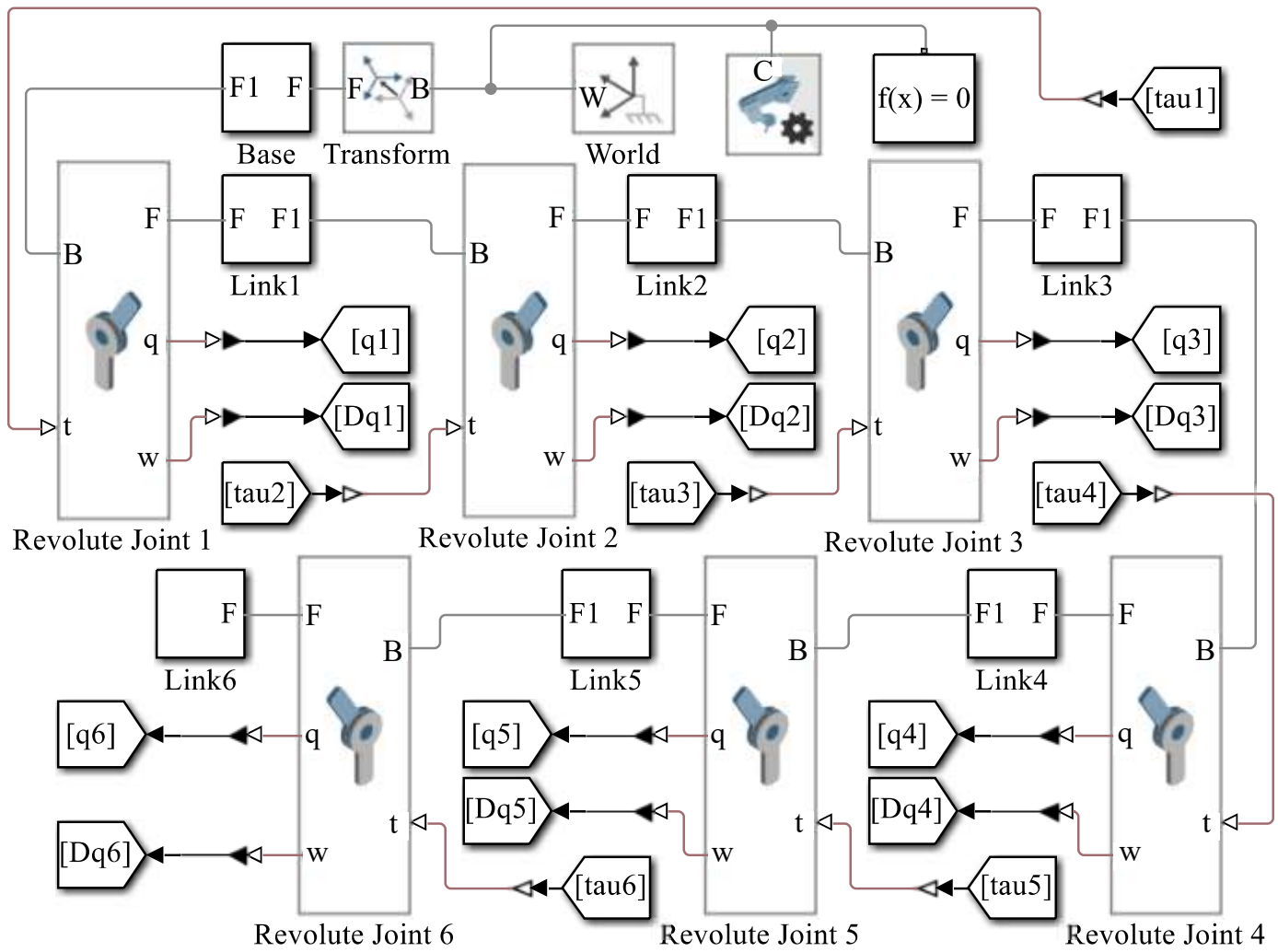

Figure 3. Simscape-based quasi-physical model of the IRB 120 robot

\section{CLASSIFICATION AND MODEL OF ACTUATOR TORQUE DEGRADATION FAULTS}

After a long time working, robots may fail caused by their parts becoming faulty. Any failure existing in the robot components will affect the behavior of the whole robot such that tasks cannot be done well as desired. The robot components are bodies, joints, actuators, transmission and gear units, hardware and software of controllers, sensors, and other mechanical and/or electrical devices. Therein, actuator failures belong in a set of joint failures. Physical constraints do not permit an actuator to provide unbounded torque and/or unlimited rate [31]-[33]. In practical activities, actuators are time-worn and may encounter many different types of faults. Faulty actuators cannot generate the required torque when they become a partial or total loss of effectiveness. The amplitude and rate bounds of actuator torque become less and less and even less than necessary values to 
accomplish tasks. The actuators of the IRB 120 robot we study in this paper are alternating-current motors. In the following subsections, three types of actuator torque degradation faults, which can occur in the $i$ th actuator as shown in Figure 4 are classified and clarified. In Figure $4, \tau_{d i}$ denotes the desired torque provided by the controller for actuator $i, \tau_{i}$ is the actual torque generated by actuator $i$.

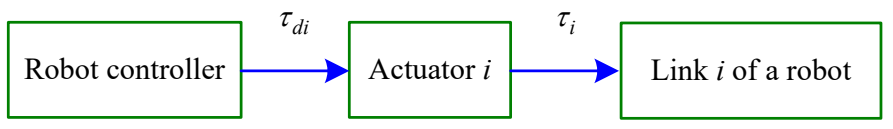

Figure 4. Block diagram for an actuator with its actual torque $\tau_{i}$ according to a desired torque $\tau_{d i}$

\subsection{Boundary degradation of torque fault}

According to the previous discussion, a specific actuator's torque is originally subject to a precise boundary. The torque boundary can vary depending on different sorts of actuators.

$$
\tau_{i \min } \leq \tau_{i} \leq \tau_{i \max }, \text { and } \tau_{i}=\left\{\begin{array}{lll}
\tau_{d i} & \text { if } & \tau_{i \min } \leq \tau_{d i} \leq \tau_{i \max } \\
\tau_{i \max } & \text { if } & \tau_{d i}>\tau_{i \max } \\
\tau_{i \min } & \text { if } & \tau_{d i}<\tau_{i \min }
\end{array}\right.
$$

where $\tau_{i \min }$ and $\tau_{i \max }$ are the original minimum and maximum torque values of actuator $i$ in regular operation, respectively. Without appropriate maintenance or even with a maintenance schedule, actuators may still degrade gradually after a period of time of operation. Besides, an abrupt, unexpected, unavoidable incident may occur. This leads to one consequence as the actuator torque boundary is tightened and become smaller.

$$
\left\{\begin{array}{l}
\tau^{\prime}{ }_{i \min } \leq \tau_{i} \leq \tau^{\prime}{ }_{i \max } \\
\tau^{\prime}{ }_{i \max }<\tau_{i \max } \\
\tau^{\prime}{ }_{i \min }>\tau_{i \min }
\end{array}, \text { and } \tau_{i}= \begin{cases}\tau_{d i} & \text { if } \quad \tau^{\prime}{ }_{i \min } \leq \tau_{d i} \leq \tau^{\prime}{ }_{i \max } \\
\tau^{\prime}{ }_{i \max } & \text { if } \tau_{d i}>\tau^{\prime}{ }_{i \max } \\
\tau^{\prime}{ }_{i \min } & \text { if } \quad \tau_{d i}<\tau^{\prime}{ }_{i \min }\end{cases}\right.
$$

where $\tau_{i \min }^{\prime}$ and $\tau_{i \max }^{\prime}$ are the new unknown minimum and maximum torque values of faulty actuator $i$. This is the first type of actuator torque degradation faults, namely boundary degradation of torque.

\subsection{Boundary degradation of torque rate fault}

Actuators may befall not only the BDT fault but also Boundary Degradation of Torque Rate fault - the second type of actuator torque degradation faults. Because of physical constraints, an actuator torque cannot fluctuate at an unlimited rate. In regular operation, the rise and fall speed of torque is limited within a boundary described as (8):

$$
\left|\frac{d \tau_{i}}{d t}\right| \leq \delta_{i}, \text { and } \frac{d \tau_{i}}{d t}=\left\{\begin{array}{ccc}
\frac{d \tau_{d i}}{d t} & \text { if } \quad\left|\frac{d \tau_{d i}}{d t}\right| \leq \delta_{i} \\
\delta_{i} & \text { if } \frac{d \tau_{d i}}{d t}>\delta_{i} \\
-\delta_{i} & \text { if } \frac{d \tau_{d i}}{d t}<-\delta_{i}
\end{array}\right.
$$

where $\delta_{i}$ is the original torque rate limit of normal actuator $i$. Robot manufacturers should concern these values in taking the most suitable motors for different bodies of a robot. Those values may be decreased after working for a long time due to previously discussed causes. The new boundary of torque rate becomes lesser as (9):

$$
\left|\frac{d \tau_{i}}{d t}\right| \leq \delta^{\prime}{ }_{i}<\delta_{i}, \text { and } \frac{d \tau_{i}}{d t}=\left\{\begin{array}{cc}
\frac{d \tau_{d i}}{d t} & \text { if } \quad\left|\frac{d \tau_{d i}}{d t}\right| \leq \delta^{\prime}{ }_{i} \\
\delta^{\prime}{ }_{i} & \text { if } \frac{d \tau_{d i}}{d t}>\delta^{\prime}{ }_{i} \\
-\delta^{\prime}{ }_{i} & \text { if } \quad \frac{d \tau_{d i}}{d t}<-\delta^{\prime}{ }_{i}
\end{array}\right.
$$

where $\delta_{i}^{\prime}$ is the actual torque rate limit of faulty actuator $i$. A low rate leads to a delay in generating an 
appropriate torque. This fault prevents actuators from reacting quickly enough; therefore, the end-effector may not track the desired trajectory perfectly.

\subsection{Proportional degradation of torque fault}

In fact, actuators also face another common fault. The third type of actuator torque degradation faults is the Proportional Degradation of Torque. The torque generated by a motor loses a certain proportion of its effectiveness.

$$
\tau_{i}=k_{i} \tau_{d i}
$$

where $k_{i}$ is the positive proportional factor of torque degradation level of faulty actuator $i$ with $0 \leq k_{i} \leq 1$. Actuator $i$ operating in fault-free state is indicated by $k_{i}=1$; and $k_{i}=0$ indicates actuator $i$ falls into total torque loss - a mentioned special case. When the total torque loss happens, the problem goes into one of the two other topics that are the locked joint and free-swinging joint issues. However, the free-swinging joint mode will not exist in a real industrial robot due to friction and gearboxes.

\section{SIMULATION RESULTS AND DISCUSSION}

In this section, the impacts of the three types of actuator torque faults on the IRB 120 robot are investigated and verified by simulations. The desired sinusoidal trajectory $q_{d}=\left[q_{1 d}, \ldots, q_{6 d}\right]^{T}$ in radian is given as (11):

$$
\begin{array}{lll}
q_{1 d}=2 \sin (\pi t) & q_{2 d}=1.5 \sin (\pi t) & q_{3 d}=\sin (\pi t) \\
q_{4 d}=2.5 \sin (\pi t) & q_{5 d}=2 \sin (\pi t) & q_{6 d}=3 \sin (\pi t)
\end{array}
$$

All parameters of robot dynamics are considered to be known and ideally precise to prevent the effects of all other causes and concentrate on the impact of only actuator torque degradation. Hence, a well-known computed-torque controller can be used for fast simulation as (12):

$$
\tau=M(q)\left(\ddot{q}_{d}+K_{d} \dot{e}+K_{p} e\right)+C(q, \dot{q}) \dot{q}+g(q)
$$

where $e=q_{d}-q$ is the tracking error vector, $K_{d}=\operatorname{diag}\left(k_{d i}\right)$ and $K_{p}=\operatorname{diag}\left(k_{p i}\right) \in R^{6 \times 6}$ are the diagonal matrices of chosen positive coefficients to ensure the robot system stability $(i=1,2, \ldots, 6)$. For having no overshoot, the controller gains are selected, such that $k_{p i}=k_{d i}^{2} / 4$. The larger $k_{d i}$ and $k_{p i}$ are, the faster response is. Although there are some upper bounds on the choice of computed-torque control parameters, in this paper these gains are chosen as $\left[k_{d 1}, k_{d 2}, \ldots, k_{d 6}\right]^{T}=[30,30,30,50,50,60]^{T}$ for the responses at the end of the robot faster than that nearby the robot base where the moved bodies are heavier. With the desired trajectory in (11), the actuator torque and the robot performance in the normal operation where all actuators work perfectly are depicted in Figures 5 and 6, respectively. For evaluating the impact of the mentioned actuator faults, the system is simulated in different cases. Every joint of the robot is subject to each of the three faults separately and independently. In each case, many tests are implemented by increasing degradation level little by little in percent. Afterwards, two critical percentages of torque losses are found out and the corresponding robot performances are compared with the normal robot performance in one figure.

When an actuator of a joint is under a fault, this fault affects not only on that joint itself but also on all other joints. However, the faulty joint's response is most strongly influenced by the fault than that of remaining joints. Therefore, only the faulty joint's response and the faulty actuator's torque are shown. In simulations, BDT and BDTR faults are set to active at 0.2 seconds, and PDT faults are activated at the beginning of simulations. For maximum accuracy, the global fixed-step solver ode14x is used with the parameters as: the solver Jacobian method is auto, the extrapolation order is 4, and the number of Newton's iterations is 5. All simulations are performed with the sample time of $0.001 \mathrm{~s}$ and rendered in a sufficient period of time to enough display the fault impact, i.e., 2 seconds (the first cycle of the desired sinusoidal signal). The detail results and discussion are given in the following subsections.

\subsection{Impact of BDT fault occurring in each actuator}

In Figure 7, a BDT fault of less than $10 \%$ occurring at joint 1 has virtually no impact on the robot response. From over $10 \%$ to $50 \%$ loss of effectiveness, the effect appears gradually, and at approximately $50 \%$ loss, the response of joint 1 deviate clearly from the desired trajectory. In Figure 8 for joint 2 and in Figure 9 
for joint 3 , the boundary of their torque reduced by just a small amount of $5 \%$ affects the robot unable to track the desired trajectory. It is understandable because joint 2 and joint 3 holding the heaviest links of the robot will be significantly affected by gravity moment. Moreover, the gravity of upper bodies also has a considerable impact on them. The responses of joint 4 and joint 6 starts to be suffered when their torque loss levels are about $20 \%$ and 30\%, respectively as shown in Figures 10 and 11. Joint 5 has better tolerance; therefore, the percent of torque loss must be approximately $50 \%$ or more to express clearly the effect on joint 5 as shown in Figure 12.
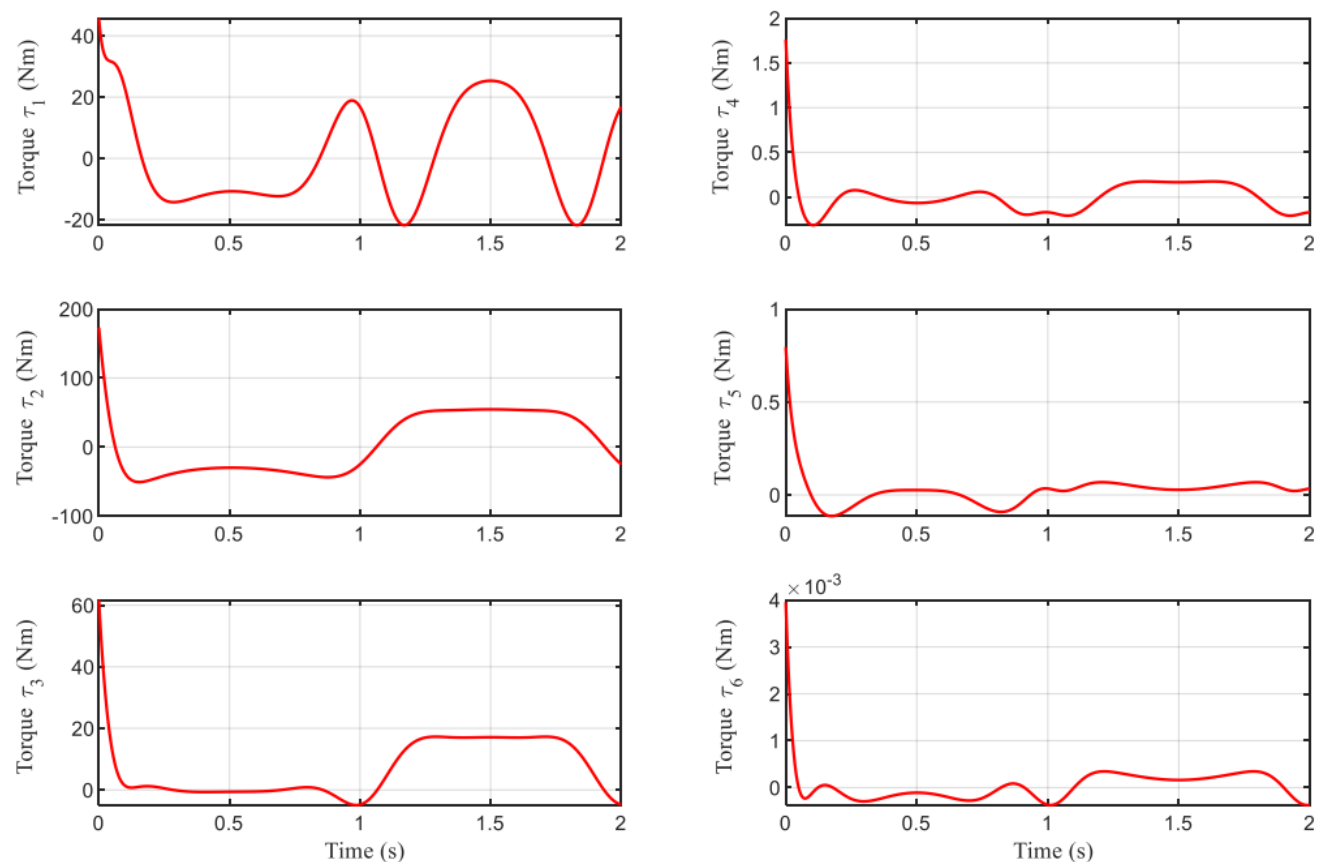

Figure 5. Actuator torque $\tau$ of the IRB 120 robot in normal operation
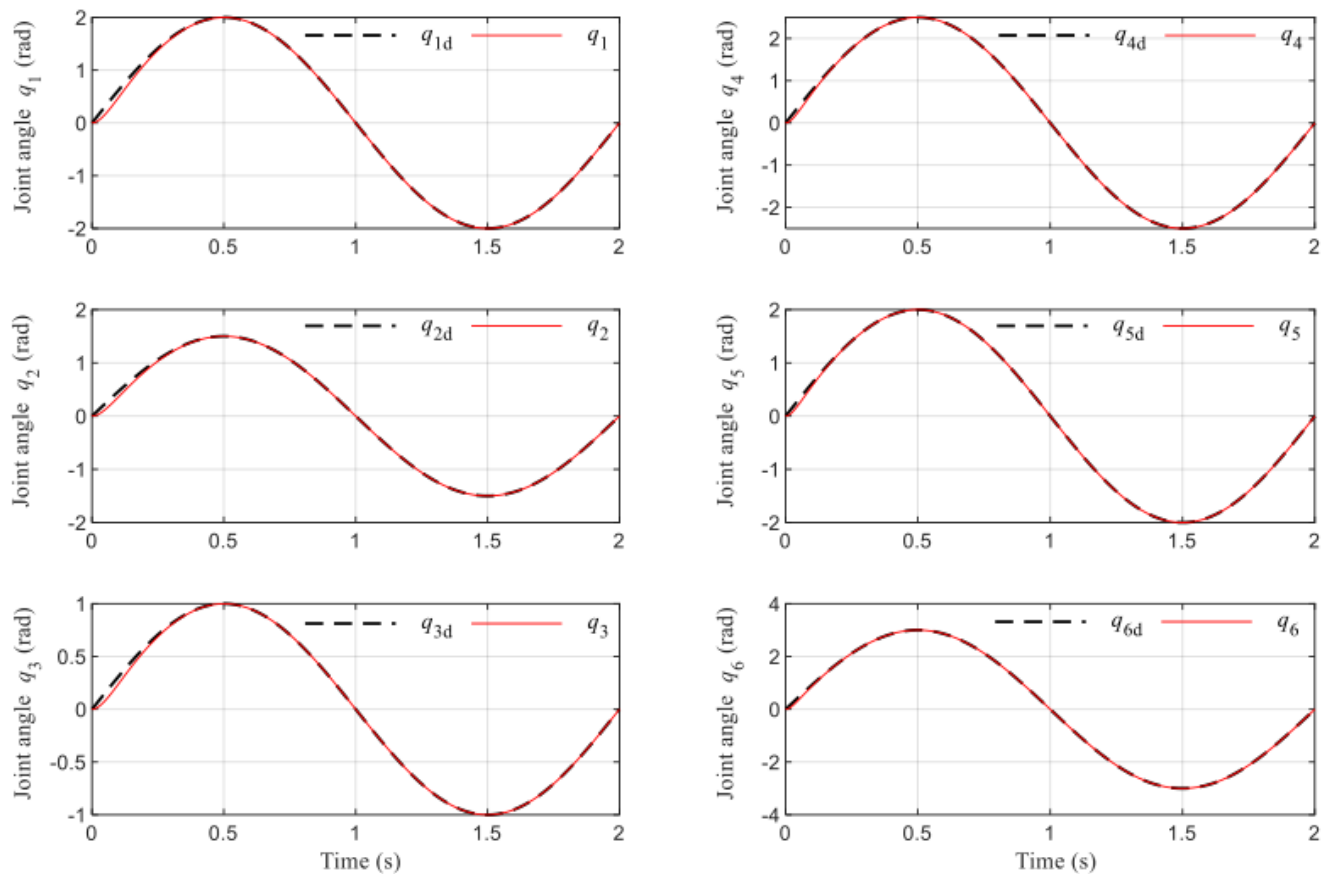

Figure 6. Desired trajectory $q_{d}$ and response $q$ of the IRB 120 robot in normal operation 

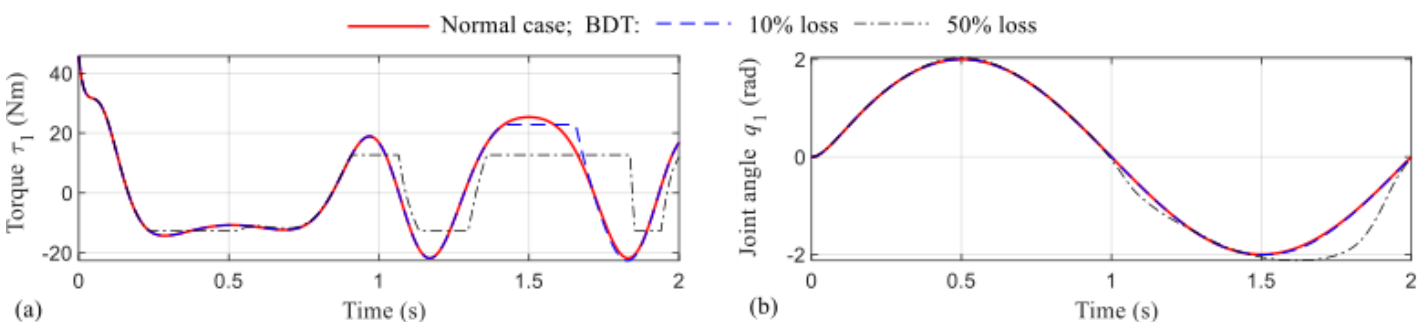

Figure 7. Impact of, (a) critical BDT levels of actuator 1 on (b) joint response $q_{1}$
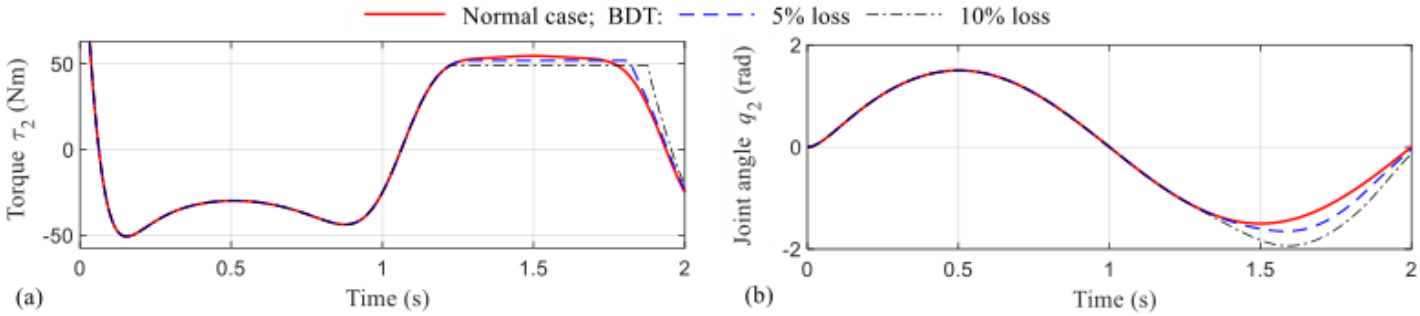

Figure 8. Impact of, (a) critical BDT levels of actuator 2 on (b) joint response $q_{2}$
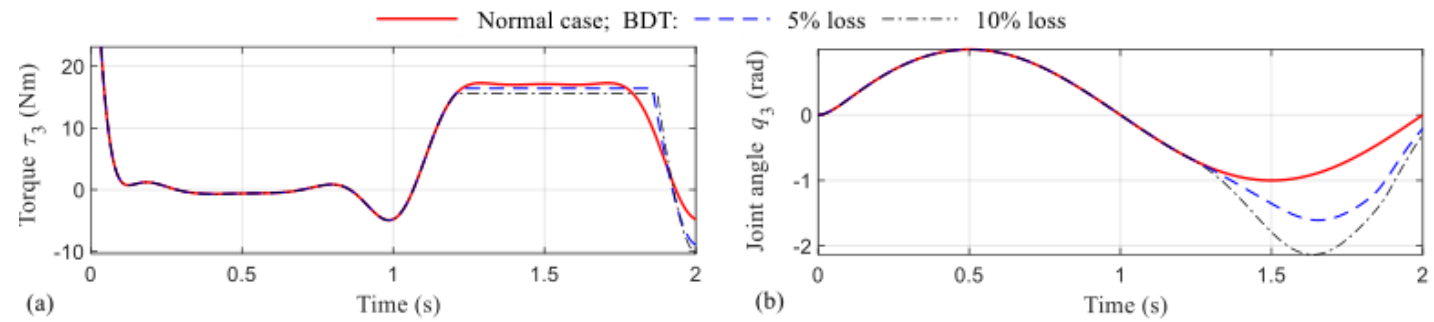

Figure 9. Impact of, (a) critical BDT levels of actuator 3 on (b) joint response $q_{3}$
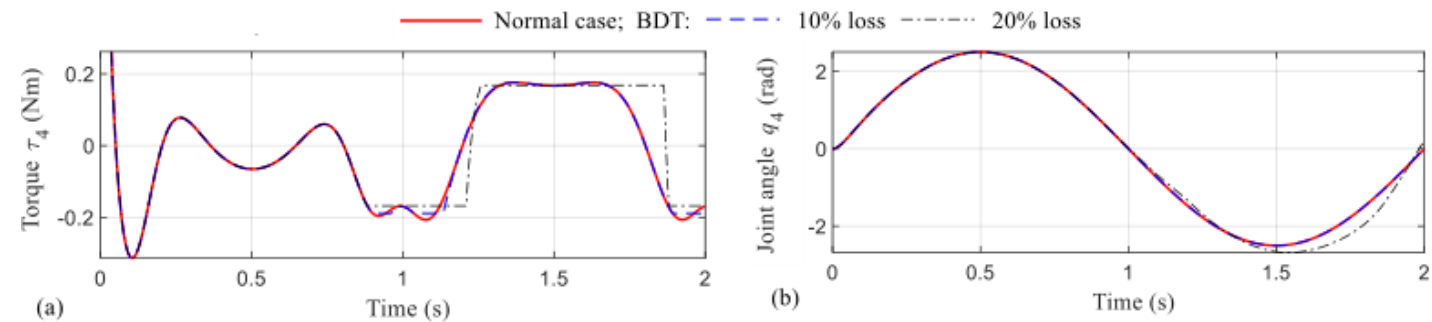

Figure 10. Impact of, (a) critical BDT levels of actuator 4 on (b) joint response $q_{4}$
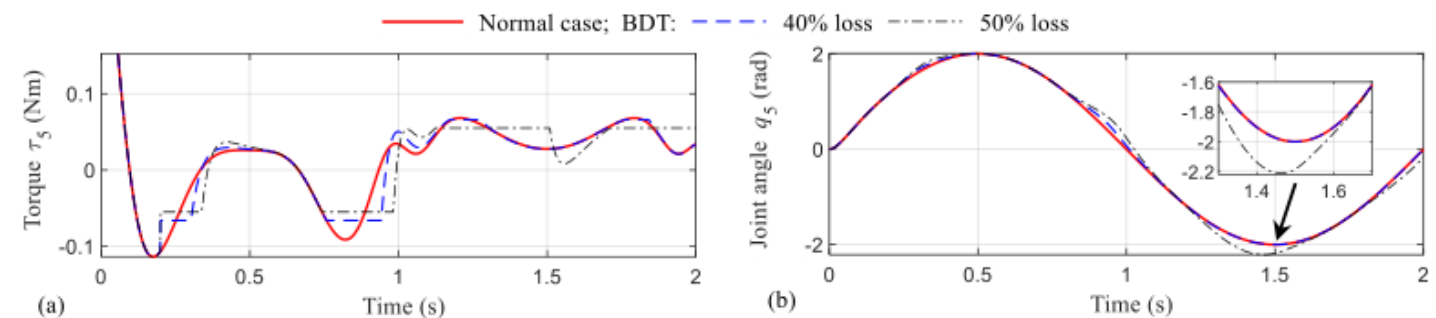

Figure 11. Impact of, (a) critical BDT levels of actuator 5 on (b) joint response $q_{5}$ 

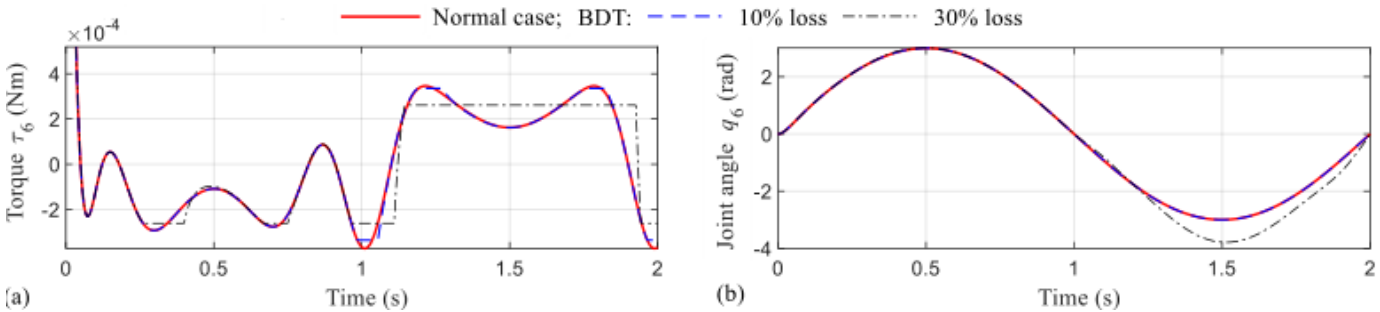

Figure 12. Impact of, (a) critical BDT levels of actuator 6 on (b) joint response $q_{6}$

\subsection{Impact of BDTR fault occurring in each actuator}

When the controller requires an actuator torque rate reaching or exceeding its capacity, the actuator can only keep its limit rate value, i.e., its constant positive upper or negative lower bound. If this happens and persists for a period of time, the rate of an actuator torque will be maintained to a constant value, and the actuator torque will increase or decrease linearly corresponding to the positive upper or negative lower bound value of its rate during that time. Resulting in the torque trajectory varies up and down in a jagged path. In Figure 13, at BDTR faults of less than 20\%, joint 1 still tries to maintain its response. However, by $25 \%$ loss, the response of joint 1 starts to be unstable and run out of control with significant value. Impacted by a lower level of torque rate loss than joint 1, the responses of joint 2 and joint 3 begin to show signs of being affected at 20\% torque rate loss in Figures 14 and 15, and will become larger at more percent of loss. Depicted in Figures 16 and 17, joint 4 and joint 5 can withstand torque rate degradation quite well up to 35\%, although the actuator torques fluctuate much more than in the normal case. Since $40 \%$ or over, their responses cannot follow the desired trajectories, and the robot starts to be uncontrollable. With weaker tolerance to BDTR faults, joint 6 can only preserve its response if torque rate loss is under $20 \%$ in Figure 18. It is clearly seen that the response of joint 6 runs far away from the desired sinusoidal signal since 25\% BDTR loss.
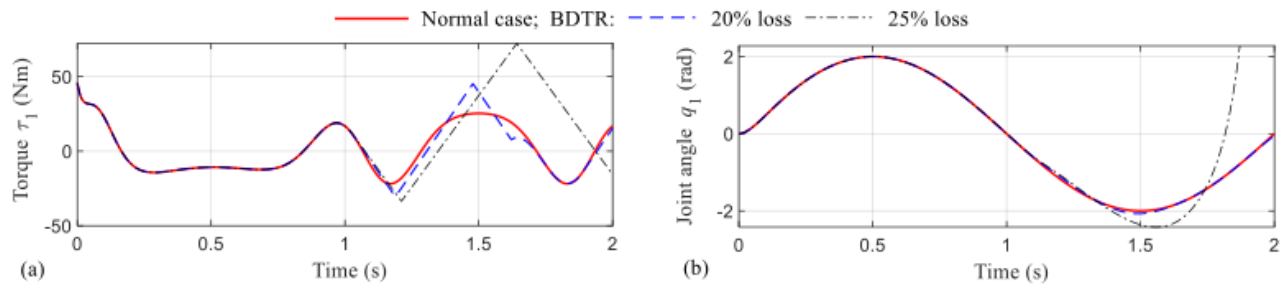

Figure 13. Impact of, (a) critical BDTR levels of actuator 1 on (b) joint response $q_{1}$
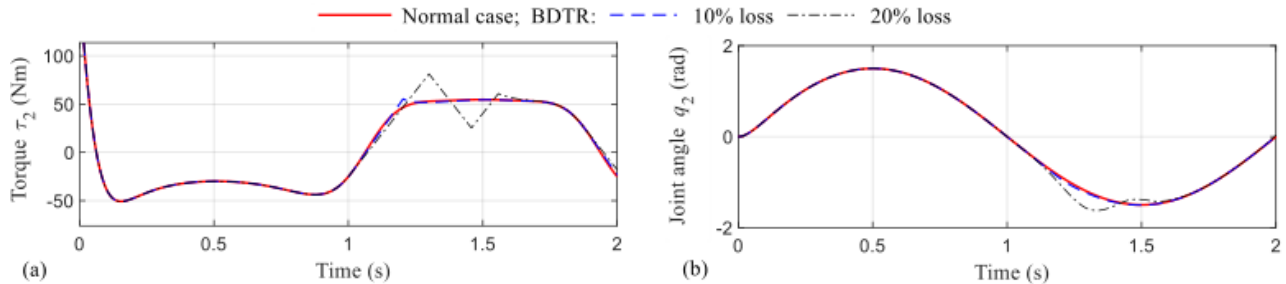

Figure 14. Impact of, (a) critical BDTR levels of actuator 2 on (b) joint response $q_{2}$
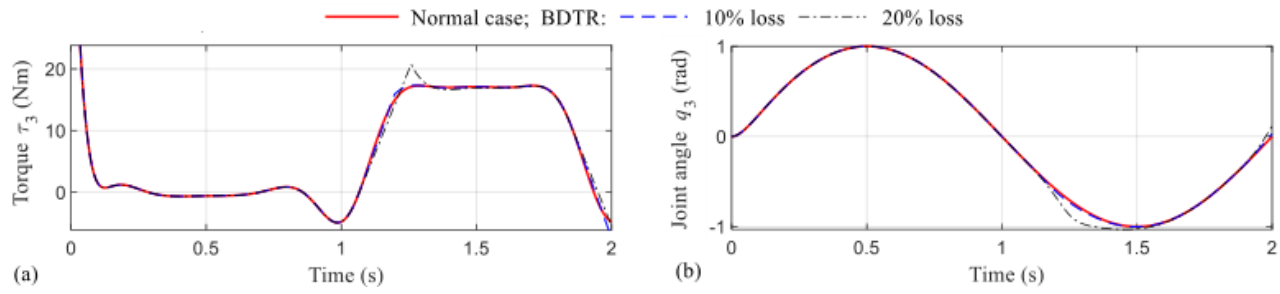

Figure 15. Impact of, (a) critical BDTR levels of actuator 3 on (b) joint response $q_{3}$ 

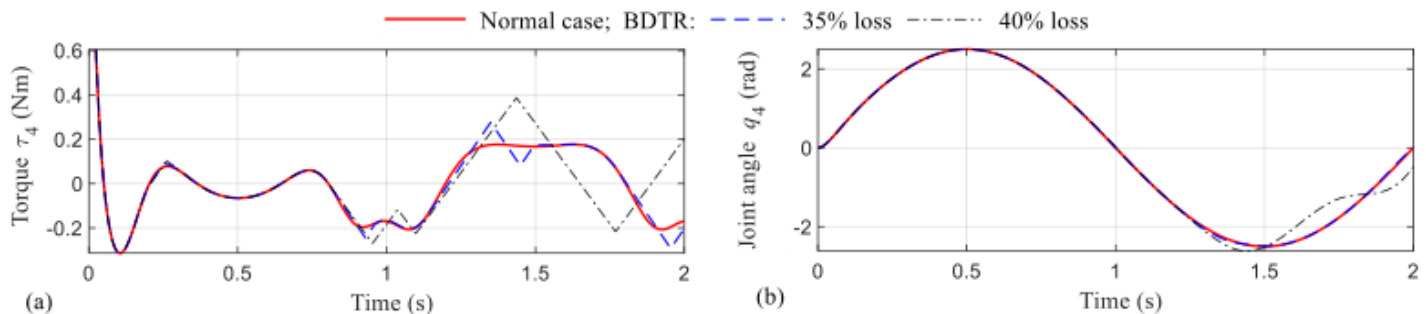

Figure 16. Impact of, (a) critical BDTR levels of actuator 4 on (b) joint response $q_{4}$
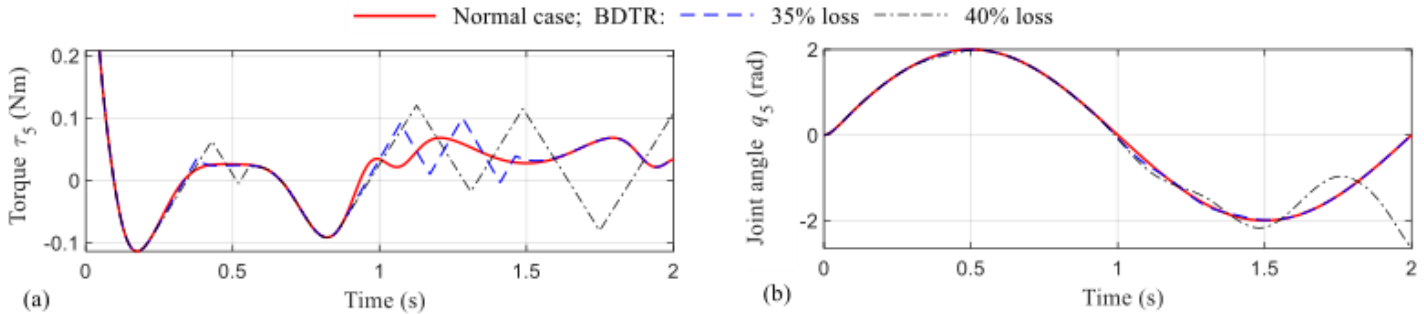

Figure 17. Impact of, (a) critical BDTR levels of actuator 5 on (b) joint response $q_{5}$
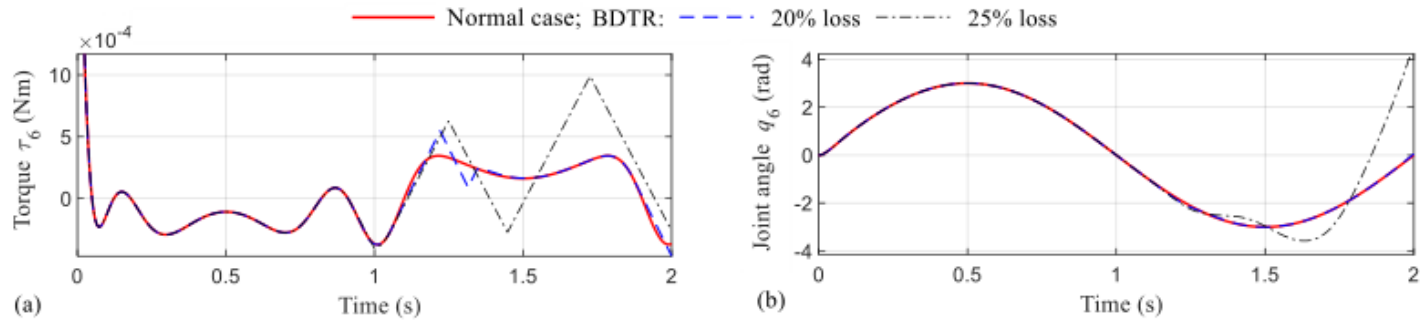

Figure 18. Impact of, (a) critical BDTR levels of actuator 6 on (b) joint response $q_{6}$

\subsection{Impact of PDT fault occurring in each actuator}

In Figure 19, the response of joint 1 keeps quite good performance at less than 10\% loss of PDT faults. The divergence from the desired trajectory grows slowly with the linear increase of torque degradation level and only shows more clearly when the loss reaches approximately 50\%. In Figures 20 and 21, same two critical cases considered at $10 \%$ and $20 \%$ loss for actuator 2 and actuator 3. The impacts on the output trajectories of both joint 2 and joint 3 start to show quite explicitly from $10 \%$ loss. The responses deviate from the desired trajectories gradually in a proportional manner. In Figures 22, 23 and 24, the responses of joints 4, 5, and 6 are affected in a similar fashion, levels of PDT loss below 10\%. These figures also show that there has almost no effect on these joints as well as the whole robot. Even if the PDT fault level of these joint actuators falls into the range of $10 \%$ to $50 \%$, their effect is quite small. Tracking errors are less than $0.1 \mathrm{rad}$ in some important points of the trajectories. In joints 1, 4, 5, and 6, when PDT faults occur below 10\%, actuator torque still quickly catches up very closely with the torque pattern of the normal case.
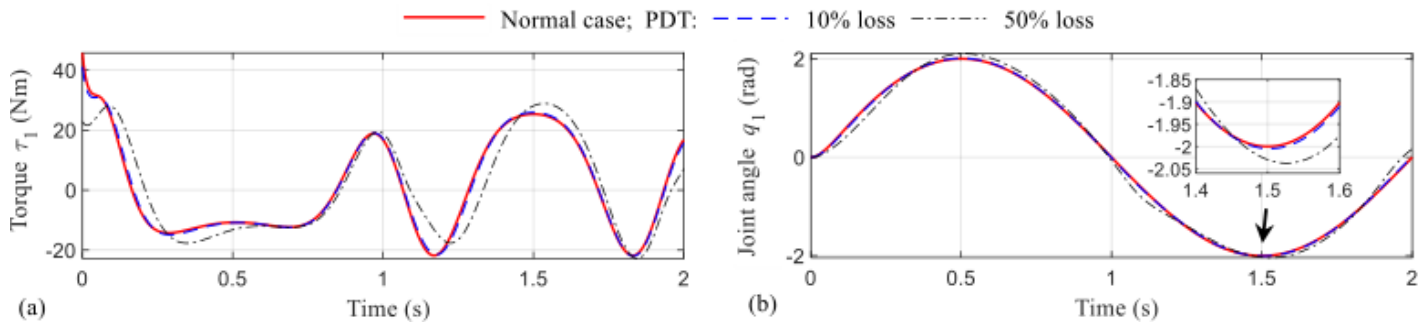

Figure 19. Impact of, (a) critical PDT levels of actuator 1 on (b) joint response $q_{1}$ 

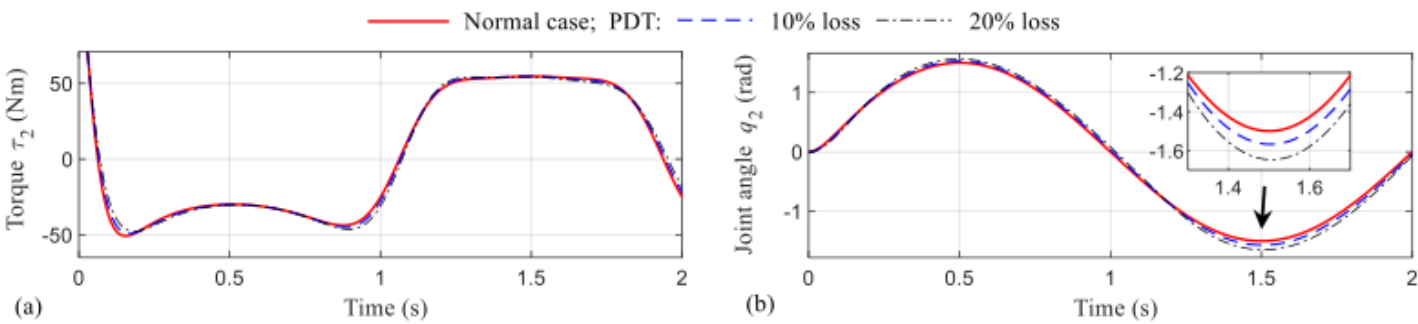

Figure 20. Impact of, (a) critical PDT levels of actuator 2 on (b) joint response $q_{2}$
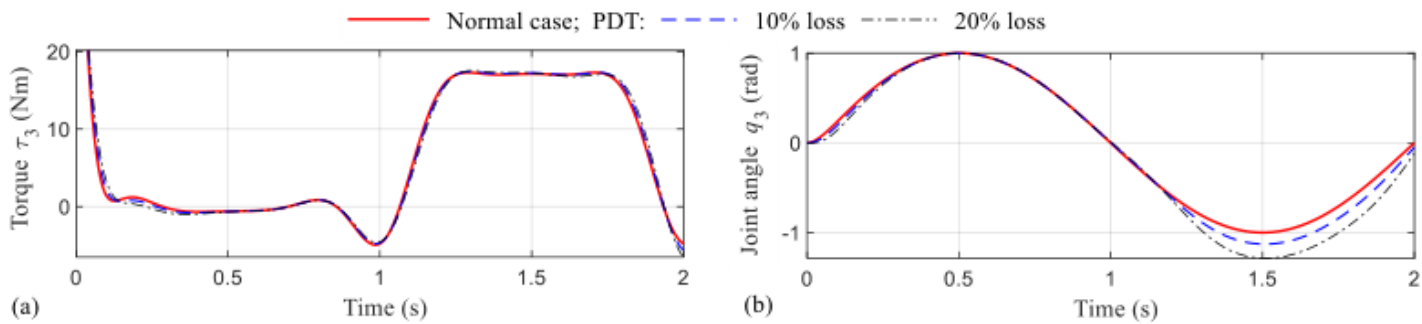

Figure 21. Impact of, (a) critical PDT levels of actuator 3 on (b) joint response $q_{3}$
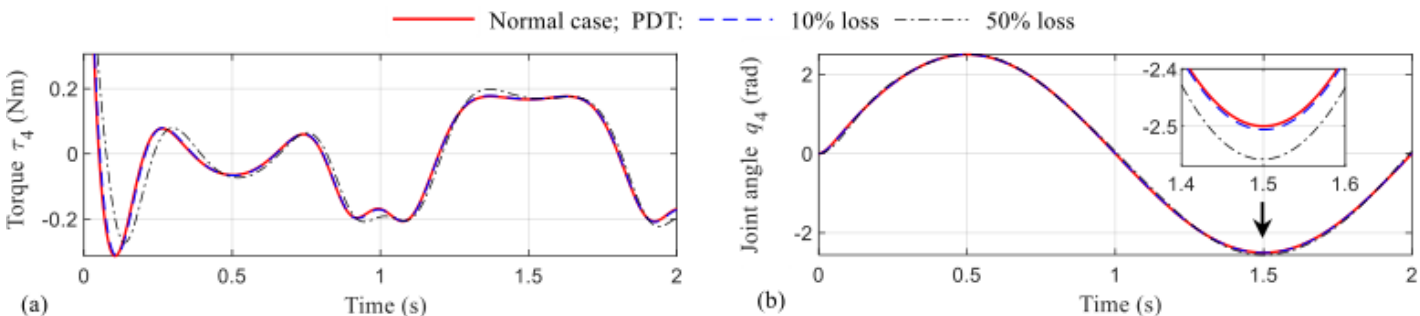

Figure 22. Impact of, (a) critical PDT levels of actuator 4 on (b) joint response $q_{4}$
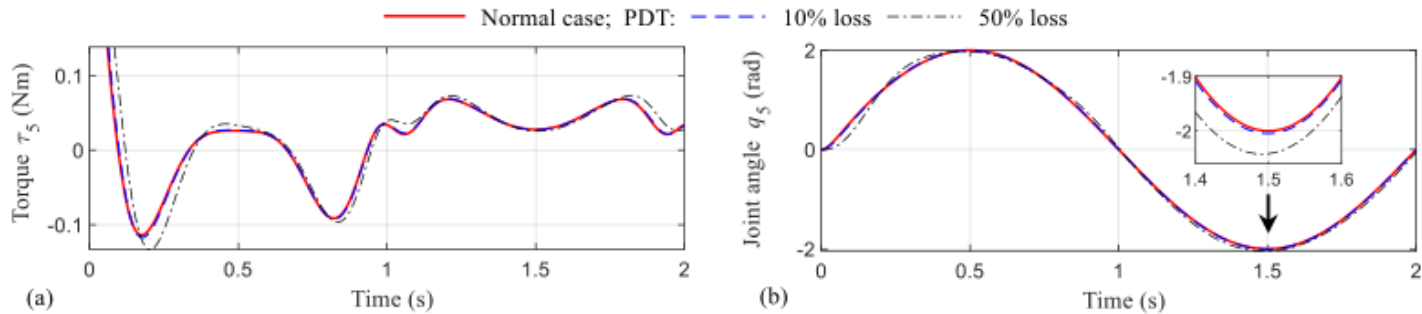

Figure 23. Impact of, (a) critical PDT levels of actuator 5 on (b) joint response $q_{5}$
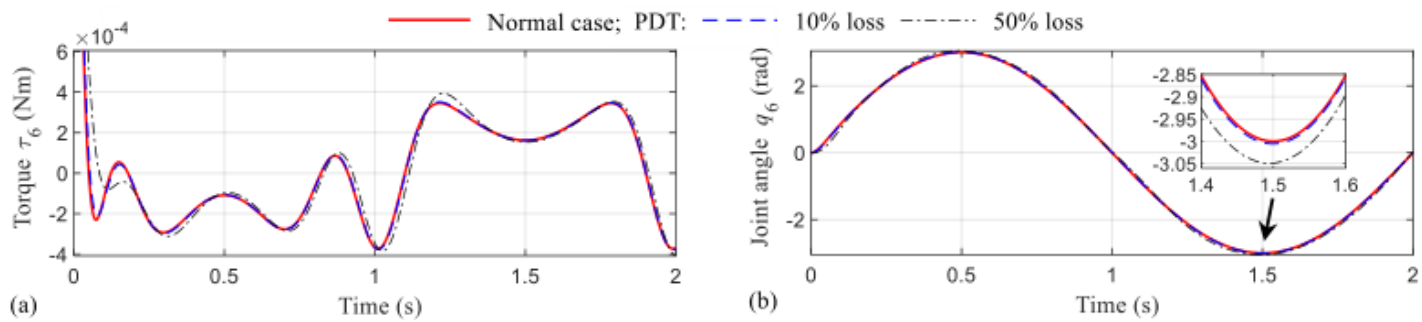

Figure 24. Impact of, (a) critical PDT levels of actuator 6 on (b) joint response $q_{6}$ 


\section{CONCLUSION}

The study analyzes the impact of actuator torque degradation faults on the performance of a typical serial robot. Three cases of actuator torque degradation faults are classified and clarified: Boundary degradation of torque, boundary degradation of torque rate, and proportional degradation of torque. Many numerical simulations are conducted using the simscape-based model of the IRB 120 robot, assures appreciable accuracy and reliability. The simulation results indicate the considerable effects of actuator torque degradation faults on the performance of the IRB 120 robot. Depending on which fault type occurring which joint, their impacts on the robot performance have different degrees. For joints more significantly affected by gravity, BDT and PDT faults will cause more severe effects. The joints which require higher torque rates will affect more influence on the robot behavior when BDTR problem occurs. Generally, it can be realized that the impact of BDTR faults is the worst whereas that of PDT faults is the most tolerable. In this study, the sinusoidal reference signals are put into the investigation. For other desired trajectories, it may still take more effort into figuring their impact in detail. The controller, which is designed before in normal situation, does not meet the control requirements when the robot is subject to most cases of actuator torque degradation faults. Hence, a suitable control strategy needs to be designed to guarantee the robot's performance when the mentioned faults happen.

\section{ACKNOWLEDGEMENT}

This research was funded by Thai Nguyen University of Technology, No. 666, $3 / 2$ street, Thai Nguyen, Vietnam.

\section{REFERENCES}

[1] J. Ghommam, N. Derbel, and Q. Zhu, New Trends in Robot Control, ser. Studies in Systems, Decision and Control 270, Singapore: Springer Singapore, 2020.

[2] T.-M. Wang, Y. Tao, and H. Liu, "Current Researches and Future Development Trend of Intelligent Robot: A Review," Int. Journal of Automation and Computing, vol. 15, no. 5, pp. 525-546, 2018, doi: 10.1007/s11633-018-1115-1.

[3] T. Dewi, S. Nurmaini, P. Risma, Y. Oktarina, and M. Roriz, "Inverse kinematic analysis of 4 DOF pick and place arm robot manipulator using fuzzy logic controller," International Journal of Electrical and Computer Engineering (IJECE), vol. 10, no. 2, pp. 1376-1386, 2020, doi: 10.11591/ijece.v10i2.pp1376-1386.

[4] T.-T. Nguyen, "Fractional-order sliding mode controller for the two-link robot arm," Int. Journal of Electrical and Computer Engineering (IJECE), vol. 10, no. 6, pp. 5579-5585, 2020, doi: 10.11591/ijece.v10i6.pp5579-5585.

[5] A.A. Jaber and R. Bicker, "Development of a Condition Monitoring Algorithm for Industrial Robots based on Artificial Intelligence and Signal Processing Techniques," International Journal of Electrical and Computer Engineering (IJECE), vol. 8, no. 2, pp. 996-1009, 2018, doi: 10.11591/ijece.v8i2.pp996-1009.

[6] W. Xu, Y. She, and Y. Xu, "Analytical and semi-analytical inverse kinematics of SSRMS-type manipulators with single joint locked failure," Acta Astronautica, vol. 105, no. 1, pp. 201-217, 2014, doi: 10.1016/j.actaastro.2014.09.003.

[7] K. M. Ben-Gharbia, A. A. Maciejewski, and R. G. Roberts, "Kinematic Design of Redundant Robotic Manipulators for Spatial Positioning that are Optimally Fault Tolerant," IEEE Transactions on Robotics, vol. 29, no. 5, pp. 13001307, 2013, doi: 10.1109/TRO.2013.2266855.

[8] C. L. Lewis and A. A. Maciejewski, "Fault tolerant operation of kinematically redundant manipulators for locked joint failures," IEEE Transactions on Robotics and Automation, vol. 13, no. 4, pp. 622-629, 1997, doi: 10.1109/70.611335.

[9] R. S. Jamisola, A. A. Maciejewski, and R. G. Roberts, "Failure-tolerant path planning for kinematically redundant manipulators anticipating locked-joint failures," IEEE Transactions on Robotics, vol. 22, no. 4, pp. 603-612, 2006, doi: 10.1109/TRO.2006.878959.

[10] H. Abdi et al., "Optimal mapping of joint faults into healthy joint velocity space for fault-tolerant redundant manipulators," Robotica, vol. 30, no. 4, pp. 635-648, 2012, doi: 10.1017/S0263574711000671.

[11] J. D. English and A. A. Maciejewski, "Fault tolerance for kinematically redundant manipulators: Anticipating free-swinging joint failures," IEEE Transactions on Robotics and Automation, vol. 14, no. 4, pp. 566-575, 1998, doi: $10.1109 / 70.704223$.

[12] J.-H. Shin and J.-J. Lee, "Fault detection and robust fault recovery control for robot manipulators with actuator failures," in 1999 IEEE International Conference on Robotics and Automation, Detroit, MI, USA, May 1999, pp. 861-866, doi: 10.1109/ROBOT.1999.772398.

[13] Z. Mu, L. Han, W. Xu, B. Li, and B. Liang., "Kinematic analysis and fault-tolerant trajectory planning of space manipulator under a single joint failure," Robotics and Biomimetics, vol. 3, no. 16, 2016, doi: 10.1186/s40638-0160048-9.

[14] Z. Mu, B. Zhang, W. Xu, B. Li and B. Liang., "Fault tolerance kinematics and trajectory planning of a 6-DOF 
space manipulator under a single joint failure," in 2016 IEEE International Conference on Real-time Computing and Robotics (RCAR), Angkor Wat, Cambodia, Jun 2016, pp. 483-488, doi: 10.1109/RCAR.2016.7784077.

[15] M. Polycarpou, "Fault accommodation of a class of multivariable nonlinear dynamical systems using a learning approach," IEEE Transactions on Automatic Control, vol. 46, no. 5, pp. 736-742, 2001, doi: 10.1109/9.920792.

[16] L. Xing et al., "Adaptive compensation for actuator failures with event-triggered input," Automatica, vol. 85, pp. 129-136, 2017.

[17] W. Wang and C. Wen, "Adaptive compensation for infinite number of actuator failures or faults," Automatica, vol. 47, pp. 2197-2210, 2011, doi: 10.1016/j.automatica.2011.08.022.

[18] Q. Yang, S. S. Ge, and Y. Sun, "Adaptive actuator fault tolerant control for uncertain nonlinear systems with multiple actuators," Automatica, vol. 60, pp. 92-99, 2015, doi: 10.1016/j.automatica.2015.07.006.

[19] A. Freddi, S. Longhi, A. Monteriù, D. Ortenzi, and D. P. Pagnotta., "Fault Tolerant Control Scheme for Robotic Manipulators Affected by Torque Faults," IFAC-PapersOnLine, vol. 51, no. 24, pp. 886-893, 2018, doi: 10.1016/j.ifacol.2018.09.680.

[20] Y. Zeng, Y. R. Xing, H. J. Ma and G. H. Yang, "Adaptive fault diagnosis for robot manipulators with multiple actuator and sensor faults," in the 27th Chinese Control and Decision Conference (CCDC). Qingdao, China: IEEE, May 2015, pp. 6569-6574, doi: 10.1109/CCDC.2015.7162006.

[21] H.-J. Ma and G.-H. Yang, "Simultaneous fault diagnosis for robot manipulators with actuator and sensor faults," Information Sciences, vol. 366, pp. 12-30, 2016, doi: 10.1016/j.ins.2016.05.016.

[22] K.O. Omali et al., "Actuator fault detection and isolation for robot manipulator using higher order sliding mode observers," Indonesian Journal of Electrical Engineering and Computer Science, vol. 17, no. 3, pp. 1150-1156, 2020, doi: 10.11591/ijeecs.v17i3.pp1150-1156.

[23] S. J. Yoo, "Actuator fault detection and adaptive accommodation control of flexible-joint robots," IET Control Theory \& Applications, vol. 6, no. 10, pp. 1497-1507, 2012, doi: 10.1049/iet-cta.2011.0508.

[24] G. Liu, "Control of robot manipulators with consideration of actuator performance degradation and failures," in 2001 IEEE International Conference on Robotics \& Automation, Seoul, Korea, May 2001, pp. 2566-2571, doi: 10.1109/ROBOT.2001.933009.

[25] "Product specification IRB 120 (Document ID: 3HAC035960-001)," 2019. [Online]. Available: https://new.abb.com/products/robotics/industrial-robots/irb-120.

[26] L. N. Truc, N. V. Quyen, and N. P. Quang, "Dynamic model with a new formulation of Coriolis/centrifugal matrix for robot manipulators," Journal of Computer Science and Cybernetics, vol. 36, no. 1, pp. 89-104, 2020.

[27] N. T. Dzung et al., "Iterative Learning Control for V-Shaped Electrothermal Microactuator," Electronics, vol. 8, no. 12, p. 1410, 2019, doi: 10.3390/electronics8121410.

[28] M. Almaged, S. I. Khather, and A. I. Abdulla, "Design of a discrete PID controller based on identification data for a simscape buck boost converter model," International Journal of Power Electronics and Drive Systems (IJPEDS), vol. 10, no. 4, pp. 1797-1805, 2019, doi: 10.11591/ijpeds.v10.i4.1797-1805.

[29] M. S. Ibbini and A. H. Adawi, "A SIMSCAPE based design of a dual maximum power point tracker of a standalone photovoltaic system," International Journal of Electrical and Computer Engineering (IJECE), vol. 10, no. 3, pp. 2912-2917, 2020, doi: 10.11591/ijece.v10i3.pp2912-2917.

[30] “IRB 120 CAD Models," 2020. [Online]. Available: https://new.abb.com/products/robotics/industrial-robots/irb120/irb-120-cad

[31] L. Notash, "A methodology for actuator failure recovery in parallel manipulators," Mechanism and Machine Theory, vol. 46, no. 4, pp. 454-465, 2011, doi: 10.1016/j.mechmachtheory.2010.11.017.

[32] L. Notash and L. Huang, "On the design of fault tolerant parallel manipulators," Mechanism and Machine Theory, vol. 38, no. 1, pp. 85-101, 2003, doi: 10.1016/S0094-114X(02)00067-8.

[33] Z. Liu, J. Liu, and W. He, "Partial differential equation boundary control of a flexible manipulator with input saturation," International Journal of Systems Science, vol. 48, no. 1, pp. 53-62, 2017, doi: 10.1080/00207721.2016.1152416.

\section{BIOGRAPHIES OF AUTHORS}

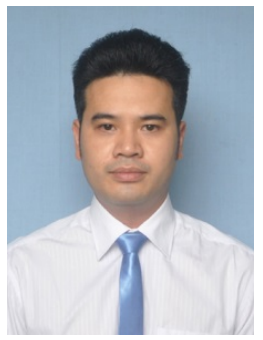

Le Ngoc Truc majored in Automatic Control and received his BS and MSc degrees from Hanoi University of Science and Technology (HUST), Vietnam, in 2006 and 2010, respectively. He has been working as a lecturer in Hung Yen University of Technology and Education since 2007. He is currently a PhD student of HUST. His researches are in fields of Control Engineering, Robotics, System Dynamics and Modeling. 


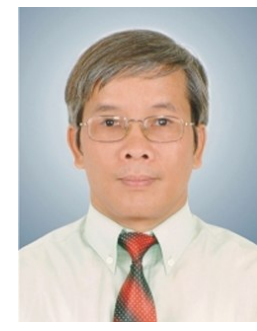

Nguyen Phung Quang received his Dipl.-Ing. (Uni.), Dr.-Ing. and Dr.-Ing. habil. degrees from TU Dresden, Germany in 1975, 1991 and 1994 respectively. Prior to his return to Vietnam, he had worked in Germany industry for many years, contributed to create inverters REFU 402 Vectovar, RD500 (REFU Elektronik); Simovert 6SE42, Master Drive MC (Siemens). From 1996 to 1998, he served as assistant lecturer of TU Dresden where he was conferred as Privatdozent in 1997. He joined Hanoi University of Science and Technology in 1999, as lecturer up to now. He is currently a professor of HUST and honorary professor of TU Dresden. He was author/co-author of more than 170 journal and conference papers; 8 books with two among them was written in German and one in English entitled "Vector Control of Three-Phase AC Machines - System Development in the Practice" published by Springer in 2008, and 2nd edition in June 2015. His researches are in fields of Control of Electrical Machines and Drives, Robot and Motion Control, Control of Power Electronics, Control of Renewable Energy Systems.

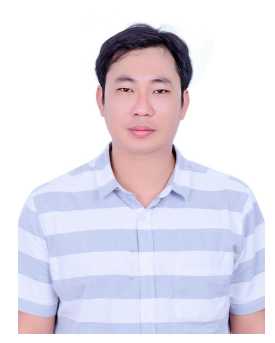

Nguyen Hong Quang is currently a lecturer at the Thai Nguyen University of Technology, Vietnam. His research interests include electrical drive systems, control systems and its applications, adaptive dynamic programming control, robust nonlinear model predictive control, motion control, and mechatronics. Further info on his ORCID: https://orcid.org/0000-0002-0433-3094. 